\title{
Soliton solution of the osmosis $K(2,2)$ equation
}

\author{
Jiangbo Zhou *, Lixin Tian \\ Nonlinear Scientific Research Center, Faculty of Science, Jiangsu University, \\ Zhenjiang, Jiangsu 212013, China
}

\begin{abstract}
In this Letter, by using the bifurcation method of planar dynamical systems, we obtain the analytic expressions of soliton solution of the osmosis $K(2,2)$ equation: $u_{t}+\left(u^{2}\right)_{x}-\left(u^{2}\right)_{x x x}=0$.
\end{abstract}

Key words: osmosis $K(2,2)$ equation; soliton; bifurcation method 1991 MSC: 35Q51, 35Q53, 37K10

\section{Introduction}

Since the theory of solitons has very wide applications in fluid dynamics, nonlinear optics, biochemistry, microbiology, geophysics and many other fields, the study of soliton solutions has become one of the important issues of nonlinear evolution equations [1]-[8].

Recently, Xu and Tian [9] investigated the osmosis $K(2,2)$ equation

$$
u_{t}+\left(u^{2}\right)_{x}-\left(u^{2}\right)_{x x x}=0,
$$

* Corresponding author. Tel.: +86-511-88969336; Fax: +86-511-88969336.

Email address: zhoujiangbo@yahoo.cn (Jiangbo Zhou). 
where the positive convection term $\left(u^{2}\right)_{x}$ means the convection moves along the motion direction, and the negative dispersive term $\left(u^{2}\right)_{x x x}$ denotes the contracting dispersion. They obtained the peaked solitary wave solution and the periodic cusp wave solution of Eq.(1.1). Unfortunately, the results are not complete. In the present Letter, we shall continue their work and obtain the smooth soliton solutions of Eq.(1.1), so that we can supplement the results of $[9]$.

The remainder of this Letter is organized as follows. In Section 2, we state the main results which are analytic expressions of the smooth soliton solutions of Eq.(1.1). In Section 3, we give the proof of the main results.

\section{Main results}

We state our main result as follows.

Theorem 2.1 For given constants $c \neq 0$ and $-\frac{c^{2}}{4}<g<-\frac{2 c^{2}}{9}$, let $\xi=x-c t$,

(1) If $c<0$, then Eq.(1.1) has a soliton solution of the form:

$$
\beta_{1}\left(\varphi_{1}^{-}\right)=\beta_{1}(\varphi) \exp \left(-\frac{1}{2} \xi\right)
$$

where

$$
\begin{aligned}
& \beta_{1}(\varphi)=\frac{\left(2 \sqrt{\varphi^{2}+l_{1} \varphi+l_{2}}+2 \varphi+l_{1}\right)\left(\varphi-\varphi_{0}^{-}\right)^{\alpha_{1}}}{\left(2 \sqrt{a_{1}} \sqrt{\varphi^{2}+l_{1} \varphi+l_{2}}+b_{1} \varphi+l_{3}\right)^{\alpha_{1}}}, \\
& \varphi_{0}^{-}=\frac{1}{2}\left(c-\sqrt{c^{2}+4 g}\right) \\
& \varphi_{1}^{-}=\frac{1}{6}\left(c+3 \sqrt{c^{2}+4 g}-2 \sqrt{c^{2}+3 c \sqrt{c^{2}+4 g}}\right) \\
& l_{1}=-\frac{1}{3}\left(c+3 \sqrt{c^{2}+4 g}\right)
\end{aligned}
$$




$$
\begin{aligned}
& l_{2}=\frac{1}{6}\left(c^{2}+6 g-c \sqrt{c^{2}+4 g}\right), \\
& l_{3}=\frac{2}{3}\left(c^{2}+6 g-c \sqrt{c^{2}+4 g}\right), \\
& a_{1}=c^{2}+4 g-c \sqrt{c^{2}+4 g}, \\
& b_{1}=\frac{1}{3}\left(2 c-6 \sqrt{c^{2}+4 g}\right) \\
& \alpha_{1}=\frac{c-\sqrt{c^{2}+4 g}}{2 \sqrt{c^{2}+4 g-c \sqrt{c^{2}+4 g}}} .
\end{aligned}
$$

(2) If $c>0$, then Eq.(1.1) has a soliton solution of the form:

$$
\beta_{2}(\varphi)=\beta_{2}\left(\varphi_{1}^{+}\right) \exp \left(\frac{1}{2} \xi\right)
$$

where

$$
\begin{aligned}
& \beta_{2}(\varphi)=\frac{\left(2 \sqrt{\varphi^{2}+m_{1} \varphi+m_{2}}+2 \varphi+m_{1}\right)\left(\varphi_{0}^{+}-\varphi\right)^{\alpha_{2}}}{\left(2 \sqrt{a_{2}} \sqrt{\varphi^{2}+m_{1} \varphi+m_{2}}+b_{2} \varphi+m_{3}\right)^{\alpha_{2}}} \\
& \varphi_{0}^{+}=\frac{1}{2}\left(c+\sqrt{c^{2}+4 g}\right) \\
& \varphi_{1}^{+}=\frac{1}{6}\left(c+3 \sqrt{c^{2}+4 g}+2 \sqrt{\left.c^{2}+3 c \sqrt{c^{2}+4 g}\right)},\right. \\
& m_{1}=-\frac{1}{3}\left(c-3 \sqrt{c^{2}+4 g}\right) \\
& m_{2}=\frac{1}{6}\left(c^{2}+6 g+c \sqrt{c^{2}+4 g}\right) \\
& m_{3}=\frac{2}{3}\left(c^{2}+6 g+c \sqrt{c^{2}+4 g}\right) \\
& a_{2}=c^{2}+4 g+c \sqrt{c^{2}+4 g} \\
& b_{2}=\frac{1}{3}\left(2 c+6 \sqrt{c^{2}+4 g}\right) \\
& \alpha_{2}=\frac{c+\sqrt{c^{2}+4 g}}{2 \sqrt{c^{2}+4 g+c \sqrt{c^{2}+4 g}}} .
\end{aligned}
$$


We shall give the proof of this theorem in Section 3. Now we take a set of data and employ Maple to display the graphs of the soliton solutions on $u-\xi$ plane.

Example 2.1 Taking $c=-2$ and $g=-0.9999$ (corresponding to (1) of Theorem 2.1), it follows that $\varphi_{0}^{-}=-1.01, \varphi_{1}^{-}=-0.979924, l_{1}=0.646667$ and $l_{2}=-0.326567, l_{3}=-1.30627, a_{1}=0.0404, b_{1}=-1.37333$, and $\alpha_{1}=$ -5.02494. We present the graph of the soliton solution in Fig.1 (a).

Example 2.2 Taking $c=2$ and $g=-0.9999$ (corresponding to (2) of Theorem 2.1), it follows that $\varphi_{0}^{+}=1.01, \varphi_{1}^{+}=0.979924, m_{1}=-0.646667$ and $m_{2}=-0.326567, m_{3}=-1.30627, a_{2}=0.0404, b_{2}=1.37333, \alpha_{2}=5.02494$. The graph of the soliton solution is presented in Fig.1(b).

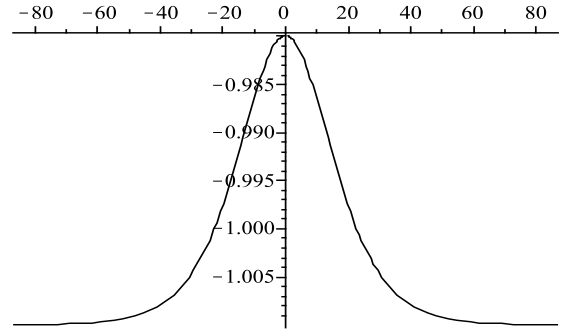

(a)

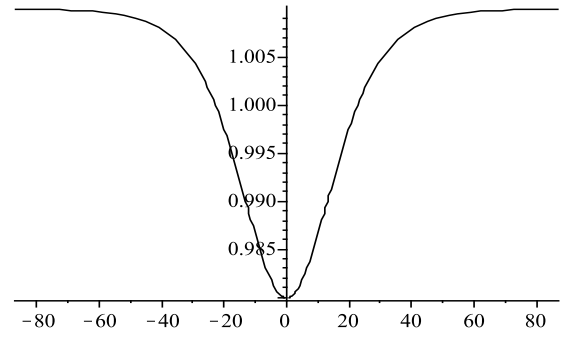

(b)

Fig. 1. The graphs of the soliton solutions. (a) $c=-2, g=-0.9999$; (b) $c=2$, $g=-0.9999$.

\section{Proof of main results}

Eq.(1.1) also takes the form

$$
u_{t}+2 u u_{x}-6 u_{x} u_{x x}-2 u u_{x x x}=0
$$


Let $u=\varphi(\xi)$ with $\xi=x-\operatorname{ct}(c \neq 0)$ be the solution of Eq.(3.1), then it follows that

$$
-c \varphi^{\prime}+2 \varphi \varphi^{\prime}-6 \varphi \varphi^{\prime \prime}-2 \varphi \varphi^{\prime \prime \prime}=0 .
$$

Integrating (3.2) once we have

$$
-c \varphi+(\varphi)^{2}-2\left(\varphi^{\prime}\right)^{2}-2 \varphi \varphi^{\prime \prime}=g,
$$

where $g$ is the integral constant.

Let $y=\varphi^{\prime}$, then we get the following planar dynamical system:

$$
\left\{\begin{array}{l}
\frac{d \varphi}{d \xi}=y \\
\frac{d y}{d \xi}=\frac{\varphi^{2}-c \varphi-g-2 y^{2}}{2 \varphi}
\end{array}\right.
$$

with a first integral

$$
H(\varphi, y)=\varphi^{2}\left(y^{2}-\frac{1}{4} \varphi^{2}+\frac{c}{3} \varphi+\frac{1}{2} g\right)=h
$$

where $h$ is a constant.

Note that (3.4) has a singular line $\varphi=0$, to avoid the line temporarily we make transformation $d \xi=2 \varphi d \zeta$. Under this transformation, Eq.(3.4) becomes

$$
\left\{\begin{array}{l}
\frac{d \varphi}{d \zeta}=2 \varphi y \\
\frac{d y}{d \zeta}=\varphi^{2}-c \varphi-g-2 y^{2}
\end{array}\right.
$$

Eq.(3.4) and Eq.(3.6) have the same first integral as (3.5). Consequently, system (3.4) has the same topological phase portraits as system (3.6) except for 
the straight line $\varphi=0$. Obviously, $\varphi=0$ is an invariant straight-line solution of system (3.6).

For a fixed $h,(3.5)$ determines a set of invariant curves of (3.6). As $h$ is varied, (3.5) determines different families of orbits of (3.6) having different dynamical behaviors. Let $M\left(\varphi_{e}, y_{e}\right)$ be the coefficient matrix of the linearized system of (3.6) at the equilibrium point $\left(\varphi_{e}, y_{e}\right)$, then

$$
M\left(\varphi_{e}, y_{e}\right)=\left(\begin{array}{rr}
y_{e} & 2 \varphi_{e} \\
2 \varphi_{e}-c & -4 y_{e}
\end{array}\right)
$$

and at this equilibrium point, we have

$$
\begin{aligned}
& J\left(\varphi_{e}, y_{e}\right)=\operatorname{det} M\left(\varphi_{e}, y_{e}\right)=-4 y_{e}^{2}-4 \varphi_{e}\left(\varphi_{e}-\frac{c}{2}\right), \\
& p\left(\varphi_{e}, y_{e}\right)=\operatorname{trace}\left(M\left(\varphi_{e}, y_{e}\right)\right)=-3 y_{e}
\end{aligned}
$$

By the theory of planar dynamical system (see [10]), for an equilibrium point of a planar dynamical system, if $J<0$, then this equilibrium point is a saddle point; it is a center point if $J>0$ and $p=0$; if $J=0$ and the Poincaré index of the equilibrium point is 0 , then it is a cusp.

In [9], $\mathrm{Xu}$ and Tian reported that when $-\frac{c^{2}}{4}<g<-\frac{2 c^{2}}{9}$, system (3.4) has two equilibrium points $\left(\varphi_{0}^{-}, 0\right)$ and $\left(\varphi_{0}^{+}, 0\right)$. (i) If $c<0$, then $\left(\varphi_{0}^{-}, 0\right)$ is a saddle point while $\left(\varphi_{0}^{+}, 0\right)$ is a center point. There is inequality $\varphi_{0}^{-}<\frac{c}{2}<\varphi_{0}^{+}<0$; (ii) If $c>0$, then $\left(\varphi_{0}^{-}, 0\right)$ is a center point while $\left(\varphi_{0}^{+}, 0\right)$ is a saddle point. There is inequality $0<\varphi_{0}^{-}<\frac{c}{2}<\varphi_{0}^{+}$.

In the parameter region: $-\frac{c^{2}}{4}<g<-\frac{2 c^{2}}{9}$, we show the phase portraits of system (3.4) in Fig.2. 


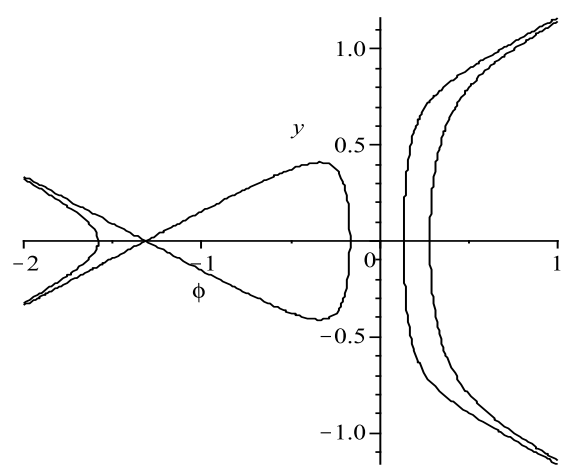

(a)

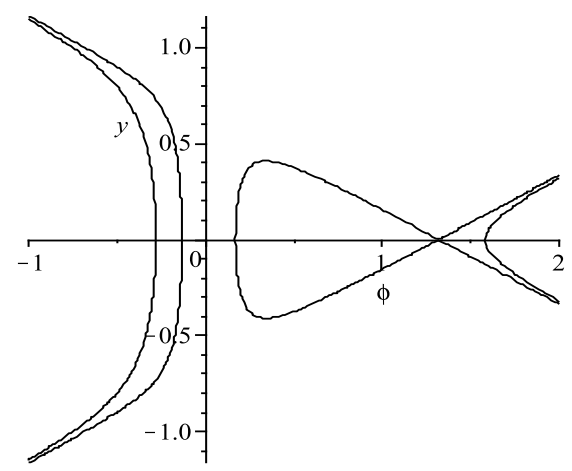

(b)

Fig. 2. The hase portraits of system (3.4) when $-\frac{c^{2}}{4}<g<-\frac{2 c^{2}}{9}$. (a) $c<0$; (b) $c>0$.

Usually, a soliton solution of Eq.(1.1) corresponds to a homoclinic orbit of system (3.4). In Fig.2(a), the homoclinic orbit of system (3.4) can be expressed as

$$
y= \pm \frac{\left(\varphi-\varphi_{0}^{-}\right) \sqrt{\varphi^{2}+l_{1} \varphi+l_{2}}}{2 \varphi} \quad \text { for } \quad \varphi_{0}^{-} \leq \varphi \leq \varphi_{1}^{-}
$$

where $\varphi_{0}^{-}, \varphi_{1}^{-}, l_{1}$ and $l_{2}$ are in (2.3), (2.4), (2.5) and (2.6), respectively. Substituting Eq.(3.7) into the first equation of system (3.4) and integrating along the homoclinic orbits, we have the expression of the solion solution as in (2.1).

In Fig.2(b), the homoclinic orbit of system (3.4) can be expressed as

$$
y= \pm \frac{\left(\varphi-\varphi_{0}^{+}\right) \sqrt{\varphi^{2}+m_{1} \varphi+m_{2}}}{2 \varphi} \quad \text { for } \quad \varphi_{1}^{+} \leq \varphi \leq \varphi_{0}^{+}
$$

where $\varphi_{0}^{+}, \varphi_{1}^{+}, m_{1}$ and $m_{2}$ are in (2.13), (2.14), (2.15) and (2.16), respectively. Substituting Eq.(3.7) into the first equation of system (3.4) and integrating along the homoclinic orbits, we have the expression of the soliton solution as in (2.11). The proof of Theorem 2.1 is completed. 


\section{References}

[1] P. Rosenau, J. M. Hyman, Phys. Rev. Lett. 70 (5) (1993) 564.

[2] A. M. Wazwaz, Appl. Math. Comput. 132 (1) (2002) 29.

[3] A. M. Wazwaz, Math. Comput. Simulat. 59 (6) (2002) 519.

[4] A. M. Wazwaz, Appl. Math. Comput. 138 (2/3) (2003) 309.

[5] Z. Y. Yan, Comput. Phys. Commun. 152 (1) (2003) 25.

[6] Y. G. Zhu, K. Tong, T. C. Lu, Chaos, Solitons and Fractals 33 (4) (2007) 1411.

[7] A. Biswas, Phys. Lett. A 372 (2008) 4601.

[8] L. Xu, Chaos, Solitons and Fractals 37 (1) (2008) 137.

[9] C. H. Xu, L. X. Tian, Chaos, Solitons and Fractals (2007), in press.

[10] D. Luo et al., Bifurcation Theory and Methods of Dynamical Systems, World Scientific Publishing Co., London, 1997. 